\title{
La Psicología del Deporte en selecciones de fútbol de Costa Rica: experiencias y reflexiones
}

\author{
Luis Diego Hernández Bonilla \\ Federación Costarricense de Futbol, Costa Rica
}

RESUMEN: El objetivo del presente artículo es exponer algunas experiencias y reflexiones adquiridas en el desarrollo de la Psicología del Deporte con selecciones juveniles masculinas de fútbol de las categorías: sub-23 (pre-olímpica), sub-20, sub-17, y sub-15 de Costa Rica. En cada una de estas selecciones, se han realizado trabajos grupales e individuales con el fin de optimizar el rendimiento y crear una fortaleza mental para superar las adversidades que presenta el alcanzar un mundial, como son las eliminatorias, pruebas físicas, o bien aspectos de carácter social como situaciones familiares, académicas, y personales por las que pasan estos futbolistas. En las competencias de alto rendimiento las exigencias no son solamente físicas, técnicas o tácticas, sino también de carácter psíquico, emocional y conductual. Para ello, se ha buscado dar respuesta a las siguientes preguntas: ¿Qué características psicológicas deben poseer estos deportistas para alcanzar el éxito? y ¿Cómo podemos entrenarlas? En el presente trabajo se comparte el reto que representa trabajar la parte mental de jóvenes deportistas talentosos, según sus características individuales, el entorno, la edad y el contexto histórico.

PALABRAS CLAVES: Psicología de rendimiento, preparación psicológica, desarrollo de talentos.

\section{Sport Psychology in Costa Rican Soccer Teams: Experiences and Reflections}

ABSTRACT: The aim of this article is to present some experiences and insights gained in the development of Sport Psychology with male youth Costa Rican national soccer team categories: U-23 (pre-Olympic), U-20, U-17 and U-15. With each of these national teams, individual and group projects have been conducted to optimize performance and to build the mental strength needed to overcome adversity arising on the road to a World Cup, such as qualifiers, physical tests, or social aspects such as family situations, academic difficulties and personal problems that most players experience. In high performance competitions, the skills demanded are not only physical, technical or tactical, but also mental, emotional and behavioral. To this end, we have tried to answer the following questions: What psychological characteristics must these athletes have to achieve success? and How can we train them? In this paper, we will explain the challenge of working on the mental aspects of these talented young athletes, according to their individual characteristics, environment, age and historical context.

KEYWORDS: Performance Psychology, psychological training, talent development.

\section{Psicologia do Esporte em equipes de futebol de Costa Rica: experiências e reflexões}

RESUMO: O objetivo deste artigo é apresentarnos algumas experiências e reflexões resultado do desenvolvimento da Psicologia do Esporte com seleções juvenis masculinas de futebol das categorias: U23 (pré-olímpico), sub-20, sub-17 e sub 15 de Costa Rica. Com cada uma dessas seleções tem-se levado a cabo trabalho individual e em grupo, a fim de otimizar o desempenho e criar uma força mental para superar a adversidade que supõe atingir uma competição global, tais como eliminatórias, provas físicas, ou aspetos de caráter social como situações familiares, acadêmicas e pessoais que passam esses jogadores. Nas competências de alto desempenho as exigências não são apenas físicas, técnicas ou táticas, mas também de caráter psíquico, emocional e comportamental. Para isso, temos procurado responder às seguintes perguntas: Quais são as características psicológicas que devem possuir esses atletas para alcançar o sucesso? e Como podemos treiná-las? Neste trabalho compartilha-se o desafio de trabalhar a parte mental dos jovens esportistas talentosos, em função das suas caraterísticas individuais, o ambiente, a idade e o contexto histórico.

PALAVRAS-CHAVE: Palavras-chave: Psicología do desempenho, formação psicológica, desenvolvimento de talentos.

Artículo recibido: 07/06/2016 | Artículo aceptado: 25/08/2016

El autor es Psicólogo. Responsable del Área de Psicología del Deporte en la Federación Costarricense de Fútbol. Presidente de ACOPDE (Asociación Costarricense de Psicología del Deporte).

La correspondencia sobre este artículo debe enviarse al autor al E-mail: Idhernandez@fedefutbol.com

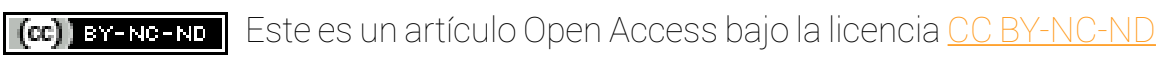


A modo de contextualización, como describe la Federación Costarricense de Fútbol (s.f. a), al referirnos a los inicios del fútbol en Costa Rica, hay que tener presente dos aspectos fundamentales. Primeramente, el país para el último cuarto del siglo XIX estaba inmerso en una economía capitalista, agro-exportadora y por ende muy ligada al mercado mundial, razón por la que se va a experimentar una influencia no sólo política-económica, sino también cultural, de los países europeos. En segundo lugar, el nivel laboral había sufrido un cambio en cuanto a la división del trabajo, en las jornadas y sobre todo en sus horarios, creando un espacio para el entretenimiento y el ocio.

Entre los años de 1873 y 1899 se crearon los primeros clubes deportivos en los cuales se practicaron varios deportes, dentro de ello el fútbol. A partir de 1899 se da una apertura para la práctica de este deporte entre los trabajadores. Fueron apareciendo grupos que lo practicaban con más frecuencia y se fue fomentando cada vez más este novedoso deporte. Los clubes deportivos de obreros fueron organizaciones en las que existían un vínculo social y un deseo de sociabilidad entre sus miembros y fue precisamente en éstos donde se originó la práctica del fútbol.

A partir de la creación de la Liga de Fútbol en el año 1921, este deporte se centralizó a nivel administrativo y competitivo. Este órgano en el año 1931 se denominó Federación Deportiva de Costa Rica; luego se llamó Federación Nacional de Fútbol y por los años sesentas cambió a Federación Costarricense de Fútbol.
Es importante destacar que el fútbol contó con el apoyo de la Iglesia y del gobierno, por lo que tuvo gran desarrollo y se convirtió en el deporte preferido de los costarricenses hasta la fecha.

Actualmente, la Federación Costarricense de Fútbol (s.f. b) la componen los siguientes órganos (ver Figura 1): asamblea, comité ejecutivo (presidente, vicepresidente, directores), secretario general, dirección de desarrollo, financiera, legal, administrativa, de comunicación y de selecciones. De esta última se desprende la unidad médica, la cual se deriva el área de Psicología del Deporte.

La detección del talento, desarrollo y entrenamiento de los jugadores se integra dentro de un proceso compuesto por las selecciones regionales, sub-15, sub-17, sub-20 y sub-23, hasta llegar a la selección absoluta (Federación Costarricense de Fútbol, s. f. c). El primer contacto que tienen los jugadores de fútbol en el camino para ser seleccionados en Costa Rica, se lleva a cabo con el programa de selecciones regionales. Este consiste en 27 selecciones regionales, compuestas por 22 jugadores cada uno debidamente observados en cada región. Seguidamente se reduce a 8 supra-regiones a lo largo y ancho del país. Para este año 2016, se observó la cifra récord de 3.500 jóvenes en 120 comunidades en lo que constituyó la primera etapa del programa. Este primer paso permite dar le opción a los jugadores para ser observados por el cuerpo técnico de las selecciones menores de Costa Rica, y de acuerdo con su criterio incorporarlos al proceso de la selección Sub-15,

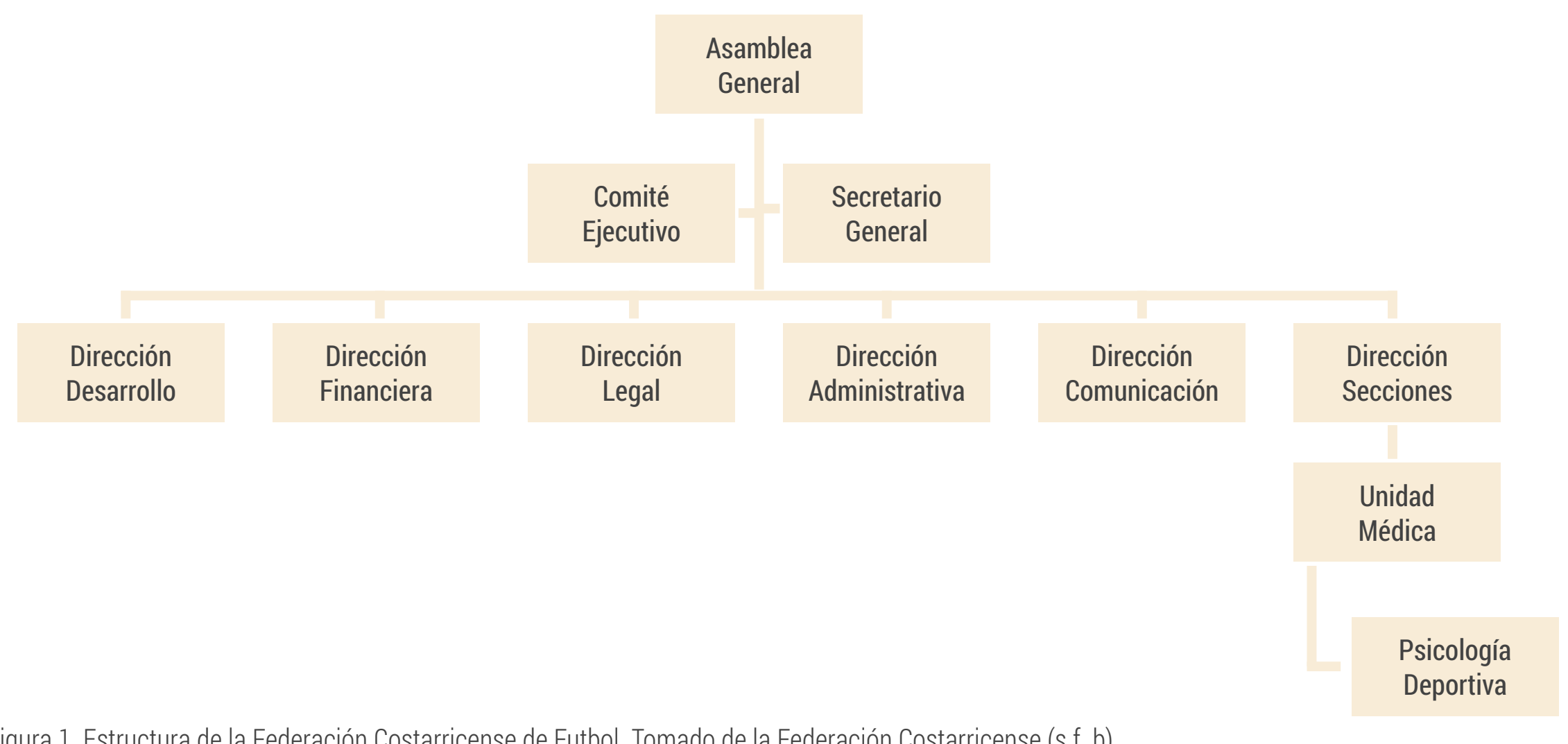

Figura 1. Estructura de la Federación Costarricense de Futbol. Tomado de la Federación Costarricense (s.f. b). 
categoría en la cual no se lleva a cabo un mundial de fútbol oficial FIFA.

Luego de pasar por programas intensos de entrenamiento y amistosos internacionales, se continúa el proceso hacia la selección sub-17, donde logran experimentar por primera vez la fase de eliminatoria y la participación en un mundial. Posteriormente luchan por un cupo para pertenecer a la selección sub-20, la cual es la antesala del futbol profesional o en muchos casos los jugadores ya han debutado en la primera división o bien han vivido pasantías en clubes importantes europeos, para después tener el privilegio de ser convocado en la selección Sub-23 o pre-olímpica, la cual cuenta con jugadores más maduros y experimentados para conseguir clasificarse a los juegos olímpicos. La meta final de este largo y difícil proceso es poder ser parte de la selección absoluta, la cual tiene como consigna clasificar y competir en un mundial, donde se parte que los seleccionados han alcanzado una consolidación importante y destacan en el medio local e internacional.

En cuanto a la labor profesional del psicólogo del deporte se inicia en febrero de 2012, siendo un puesto sin antecedentes en la federación. Previo a la inserción de este especialista, cuando se creía conveniente trabajar esta área, lo hacían con los llamados "motivadores", coach o bien con psicólogos clínicos con gran voluntad, pero sin formación específica ni experiencia en el campo de la Psicología del Deporte.

Básicamente, la labor se centra en el ámbito del alto rendimiento, cuyos objetivos son el desarrollo del talento deportivo, la mejora del rendimiento y el logro de resultados (García-Naveira, 2010). La población deportiva con la que se trabaja son los deportistas, entrenadores, preparadores físicos, coordinadores y directivos, servicios médicos, padres, árbitros, etc. (Cantón, 2010). Para ello, las funciones que se realiza son de intervención, evaluación, asesoramiento y orientación, formación e investigación (COP, 1998), integrado en un Programa Integral Deportivo (PID) en el cual se atienden diferentes pilares que sustentan un desarrollo óptimo del deportista, como son el deporte (fútbol), los estudios, la educación-formación, las familias y relaciones sociales (García-Naveira y Jerez, 2012). Partiendo de estas cuestiones, la labor del psicólogo del deporte debe de cumplir al menos tres aspectos básicos: planificar, informar y coordinar su labor con el resto de profesionales (Cox, 2008).

Destacar que en este momento se cuenta con un único psicólogo del deporte a cargo del área mental de los seleccionados. Debido a esta limitación y las exigencias propias de la actividad, la jornada laboral no se apega al modelo tradicional de 8 horas por día, sino que en muchos casos esta se extiende por la vasta demanda de las diferentes selecciones. Además, los fines de semana y feriados son aprovechados por los entrenadores para para llevar a cabo entrenamientos, giras, concentraciones y partidos amistosos. En definitiva, se podría decir que el horario de trabajo es muy flexible y sometido a cambios e imprevistos, por lo que es importante la adaptación del psicólogo del deporte a esta circunstancia.

En cuanto a los recursos físicos, se cuenta con un despecho privado, debidamente acondicionado para poder atender a los seleccionados, padres de familia, cuerpos técnicos, etc. Sin embargo, el trabajo del psicólogo del deporte no se limita al despacho, sino que se desplaza al campo, para poder observar las conductas en el hábitat natural del futbolista, el terreno de juego (García-Naveira y Jerez, 2012; Roffé, 2008).

En cuanto a las poblaciones que se le brindan atención, se encuentran principalmente las selecciones menores masculinas (sub-15, sub17, sub-20 y Sub-23) y selecciones femeninas (absoluta, sub-15, sub-17, sub-20 y sub-23), aunque también se colabora con selección absoluta futbol sala y selección absoluta futbol playa, y recientemente arbitraje, secciones que también integran la federación.

La estructura básica de abordaje a nivel psicológico se clasifica en tres tipos: prevención, intervención y mantenimiento de habilidades psicológicas deportivas. En cualquiera de los tres casos, se trabaja bajo la premisa de optimización de rendimiento, sea interviniendo en la esfera deportiva, familiar, personal o académica.

Para desarrollar el trabajo se les acompaña a los partidos amistosos, eliminatorias y mundiales (siempre y cuando el entrenador lo considere necesario). Al ser un profesional con varias selecciones, la labor del psicólogo del deporte se centra en la selección que está más próxima a la competición, sin abandonar las demás selecciones, ya que se lleva un monitoreo constante directo (observación de campo, sesiones individuales y grupales, etc.) o indirecto (a través de reuniones con el entrenador, asistente, preparador físico, etc.).

Partiendo de lo expuesto con anterioridad, el objetivo del presente trabajo consiste en presentar al lector un resumen de la experiencia profesional (más de 4 años) del psicólogo del deporte en la Federación Costarricense de Fútbol con las categorías menores.

\section{Experiencia profesional}

En una eliminatoria, y aún más acentuado, en un campeonato mundial, los ojos del país están encima de la selección de futbol, sea cual sea la categoría. Los éxitos a nivel deportivo, tienen un impacto significativo en la sociedad desde el punto de vista anímico, comportamental, económico, relacional, entre otros. Los jugadores al percatarse de esto y sabien- 
do que el mundo del deporte se alimenta de triunfos y hoy en día es fríamente resultadista, muchas veces se convierte en una gran presión para ellos. El hecho de pertenecer al grupo élite de futbolistas elegidos para representar a su país conlleva a una sana responsabilidad y una gran vitrina para lograr alcanzar el punto más alto en su carrera deportiva.

Para cumplir con dicha tarea y rendir al máximo nivel, deben de atender tanto a la parcela personal como deportiva, así como el desarrollo de herramientas psicológicas para utilizar en los momentos de mayor exigencia física y mental (Cox, 2008). Para ello se realizan sesiones individuales y grupales debidamente agendadas, con el consentimiento del entrenador, los padres (ya que son intervenciones con menores de edad) y por supuesto con la disposición de cada jugador.

En estos espacios privados, se pretende primeramente crear un vínculo de empatía, de confianza entre psicólogo-futbolista. Logrando esto, se allana el camino para la intervención posterior. Es importante tener en cuenta la categoría con la que se está trabajando, para así elegir bien el vocabulario que se utiliza, la duración de la sesión, los temas de conversación, etc.

A nivel individual, el objetivo de estas sesiones es que el futbolista entienda que tiene un espacio para poder trabajar el aspecto que quiera o bien se le haya recomendado (e.g., por el entrenador), ya sea para el entrenamiento, la competición o su vida en general. Habitualmente se llevan a cabo un mínimo de 3 sesiones y un máximo de 10 por jugador. Estas sesiones suelen tener una duración entre los 30 a 60 minutos. Pueden realizarse en el despacho o bien al finalizar la sesión de entrenamiento cerca del terreno de juego. Muchas intervenciones son agendadas pero también pueden ser emergentes. Las sesiones individuales que vienen derivadas por parte del entrenador, en algunos casos por recomendación de alguna comisión (comisión de selecciones, comisión de arbitraje, junta directiva, etc.) son de carácter obligatorio. Sin embargo, también se acuerdan sesiones por

Tabla 1. Áreas y temas de intervención individual

\begin{tabular}{llll}
\hline Personal & Grupal & Familia & Académico \\
\hline Autoconfianza & Subgrupos & Divorcios & Deserción escolar \\
\hline Autoestima & Liderazgo & $\begin{array}{l}\text { Inestabilidad } \\
\text { financiera }\end{array}$ & $\begin{array}{l}\text { Bajo rendimiento } \\
\text { académico }\end{array}$ \\
\hline Autoconcepto & Claridad del rol & $\begin{array}{l}\text { Abondo } \\
\text { de padre }\end{array}$ & $\begin{array}{l}\text { Poco apoyo } \\
\text { familiar }\end{array}$ \\
\hline Carencia afectiva & Aceptación del rol & \\
\hline Control emocional & Cumplimiento del rol & \\
\hline Concentración & Cohesión & \\
\hline
\end{tabular}

solicitud del mismo seleccionado o bien por acercamiento del propio psicólogo, por lo que tendría un carácter opcional.

Como referencia, los temas que se suelen tratar en las sesiones individuales con los seleccionados costarricenses de categorías menores, incluye la parcela personal, grupal, familiar y académica (Ver Tabla 1).

Por otra parte, se llevan a cabo sesiones grupales y talleres formativos que se suelen planificar en conjunto al cuerpo técnico (e.g., mejora de la comunicación dentro del campo o actividades para que los jugadores se conozcan mejor). Se elabora un mesociclo en donde se contemplan al menos dos sesiones al mes con el equipo y se elige qué variables se desarrollarán teniendo en cuenta la edad, antecedentes (derrotas, triunfos), competición por afrontar, rival, jugar de local o visitante, entre otras cuestiones. El número de sesiones puede variar según la necesidad de la selección o bien si alguna situación inesperada lo requiere.

En cuanto a la participación en las concentraciones, fases eliminatorias y mundiales, el psicólogo del deporte se encuentra en el momento cumbre donde todo lo trabajado con los seleccionados es sometido a prueba. Generalmente cuando se acompaña a las competiciones, se asigna un tiempo de 30 minutos de trabajo psicológico eligiendo bien qué días son los oportunos, para realizar alguna charla o actividad. Los espacios "libres" o "tiempos muertos", aunque son pocos, son buenos para poder conectar con el futbolista de una manera más natural y distendida, ya que son más permeables y están más predispuestos en general.

Además, se debe de potenciar las habilidades de adaptación al lugar, al clima, la comida, el idioma, etc. El psicólogo puede colaborar en aumentar los niveles de tolerancia y fortaleza del equipo, dentro y fuera de la cancha, ya que al cabo de unos días, la monotonía de comidas, costumbres desconocidas que tiene el compañero de cuarto, o bien resultados no deseados, empieza a modificar el entorno en un ambiente más áspero. De igual forma, las interacciones entre compañeros de cuerpo técnico pueden sufrir alguna fricción, en el que la labor del psicólogo del deporte se centraría en mantener la estabilidad relacional entre todas las partes.

Un factor a tomar en cuenta es el número de días que se estará en concentración, si esta es abierta (duermen en sus casas) o cerrada (duermen en el hotel), si es en el país o fuera de él, si se encuentra en un país con una cultura similar a la propia o no, si se compite en un torneo amistoso o se luchan por puntos, si los resultados se están dando o no, si es de las primeras salidas del país o ya tienen muchos minutos internacionales acumulados, etc. En definitiva, entran en juego diferentes aspectos a tener presentes, en el que una buena gestión facilitará acercarse a los objetivos planteados. 


\section{El trabajo con el deportista}

Siguiendo con el Programa Integral Deportivo (García-Naveira y Jerez, 2012) se entiende que el aparato psíquico del seleccionado costarricense, se trabaja en cuatro áreas básicas: deportiva, familiar, social y académico. Se procede a obtener una ficha personal de cada jugador en las cuatro áreas mencionadas, y para ello se realiza un proceso de evaluación. Se trata de aportar una serie de datos para conocer al deportista conductualmente y/o como acción dentro de un proceso de intervención (Roffé, 2009). Por ejemplo, se realizan entrevistas con los seleccionados (estructurada y semi-estructurada), se aplica una batería de test (e.g., CSAI-2, CSI, NEO-FFI, etc.), y se realiza observación de campo donde se lleva una bitácora según el objetivo planificado, siendo estas las metodologías de evaluación más frecuentes. Posteriormente se elabora un informe desde la confidencialidad con las fortalezas y áreas de mejora del deportista, además del plan de trabajo según sus necesidades.

El entrenamiento mental se diseña procurando que sea a la medida de las necesidades del futbolista o equipo. Durante la intervención se monitorea que se estén cumpliendo los objetivos inicialmente planteados para competir al más alto nivel. Se parte de que el deporte per se, contribuye al desarrollo completo de la persona, a nivel físico, psicológico y social (Clarke, Smith y Thibault, 1994, citado en Miller y Kerr, 2002; Cox, 2008; Weinberg y Gould, 2010). Por lo que, además de proporcionar experiencias para mejorar a nivel físico y técnico, el deporte debe potenciar el desarrollo de habilidades psicológicas y conductas éticas del seleccionado.

Esfera deportiva. En la esfera deportiva, por medio de sesiones individuales se intenta que el deportista descubra cuáles son sus fortalezas y cuáles son sus áreas de mejora a través de diferentes actividades y autorregistros (e.g., realizando una lista de situaciones que pueden afectar al deportista en el entrenamiento, competición y/o a nivel extra deportivo (académico, familia, salud, economía, etc). Siguiendo a Díaz-Morales y García-Naveira (2001), una manera de intervenir individualmente, es conociendo qué tipo de metas se plantean los deportistas y cómo las valoran. Las metas nos darán luz de cuáles son las fuentes de motivación del deportista. Seguidamente se trabaja bajo un esquema básico para potenciar la fortaleza mental, que se le denomina las 4 C's (Marí, 2011): compromiso, confianza, concentración y cohesión (ver Figura 2). Se explica a los futbolistas en qué consisten estas variables y se eligen cuáles son las prioritarias por trabajar según sus necesidades.

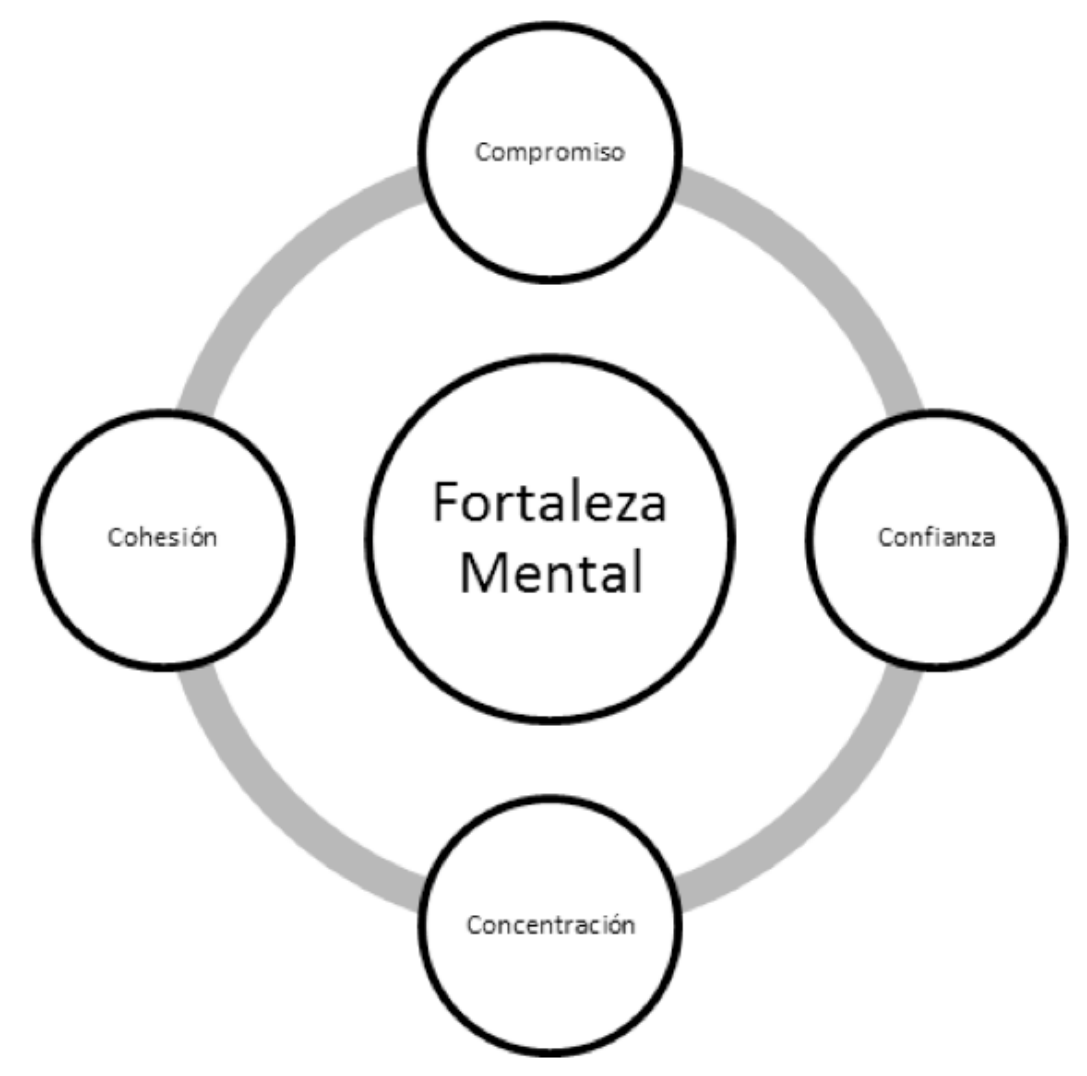

Figura 2. Esquema básico de intervención psicológica-deportiva. Adaptado de "Aprender de los campeones" por P. Marí, 2011

Lo primero que se debe determinar es si el seleccionado necesita un abordaje de intervención, es decir, carece de una habilidad básica y se le implementa de inmediato un trabajo para cubrir su necesidad. El segundo abordaje es de mantenimiento, que se refiere a que posee habilidades psicológicas en buen estado, por lo que se procede a dar contención para que pueda mantenerlos en ese nivel. El tercer abordaje es de prevención, que hace referencia al facilitar, promover e incorporar estrategias mentales que les son útiles para afrontar adversidad en el deporte o en sus vidas cotidianas. El plan de prevención se desarrolla a través de charlas, talleres y sesiones grupales de trabajo en equipo para fortalecer sus estrategias psicológicas básicas como lo son: visualización, manejo del estrés, diálogo interno, autocontrol, etc.

Esfera Familiar. En la esfera familiar, se pretende conocer a través de sesiones individuales la composición familiar, es decir, cuántos y cuáles son los miembros de su núcleo familiar, cómo percibe a cada uno de estos miembros, con quien se identifica más, con quien menos, si considera que le apoyan en el futbol o no, la escolaridad y ocupación de los padres de familia, las edades, si han practicado deporte y a qué nivel, la afinidad, confianza y grado de comunicación que existe en a dinámica familiar.

En ocasiones hay padres que se encuentran en proceso de divorcio, y este repercute en el rendimiento del futbolista, o bien hay estabilidad en las relaciones y por ende se refleja en la cancha. Se opta también por realizar charlas grupales 
a los padres de familia, para que puedan conocer las competencias que requiere ser padre de un deportista de alto rendimiento, se les facilita recomendaciones sobre el manejo del triunfo o la derrota, técnicas de retroalimentación, entre otros.

Por otra parte, se ha certificado que algunos niños y niñas, con una presión excesiva por parte de sus padres y madres, tienden a practicar la actividad deportiva como un deber más que entenderla como una forma de diversión (Gould y Petlichkoff, 1988; Holt y Dunn, 2004). Este tipo de niños y niñas, que entienden el deporte como un deber, son los que tienen más riesgo de abandonar la actividad deportiva. En la federación, se procura tener una relación cercana con los padres de familia, a través de reuniones organizadas periódicamente (trimestrales), llamadas telefónicas o bien citándoles a una sesión de despacho cuando sea conveniente. Generalmente previo a una gira o participación internacional se les convoca para brindarles los detalles del viaje y las reglas en cuanto a los mecanismos de comunicación con los hijos durante la gira, la administración del dinero que les dan a los chicos, cuido de cosas de valor que porten, etc.

Un dato interesante a tomar en cuenta según los datos reunidos en las entrevistas individuales del 2014-2015, es que un $70 \%$ de la población de seleccionados de la categoría sub-17 y sub-15 se encontraba en un núcleo familiar donde carecen de una figura paterna. Es decir, se encuentran vacíos afectivos importantes, a su vez, desarrollan un rol de "co-responsable del hogar" en donde reconocen el esfuerzo extremo de su madre y familiares cercanos para suplir las necesidades básicas (techo, comida, vestido) y esto le es combustible para luchar al máximo para llegar a brillar a nivel nacional e internacional, con el afán de brindarles una mejor calidad de vida a su familia.

Esfera Académica. Se parte de la premisa que la familia y los clubes donde pertenecen los seleccionados son los responsables directos de la formación académica. La federación como un agente más que interactúa en la formación del jugador, facilita a través de convenios con instituciones académicas públicas y privadas la posibilidad que el seleccionado este cursando las materias según su edad. Está claro que el desarrollo cognitivo y social que proporciona el colegio, universidad o cualquier centro, contribuye en el rendimiento deportivo de forma indirecta. Los deportistas para lograr pertenecer a la élite en la pre-adolescencia, adolescencia y adultez temprana, necesitan que la entidad académica les apoye en flexibilizar horarios, reponer exámenes, etc. Los padres de familia juegan un papel vital en esta tarea.

El psicólogo del deporte colabora en esta área con el seleccionado, identificando cuál es su estilo de aprendizaje, acompañamiento en cómo gestiona su tiempo de estudio, de ocio, de tareas, etc., conociendo cuál es su materia fa- vorita, cuál es la menos favorita, si se encuentra estudiando en institución pública o privada, si ha reprobado materias o años, si es destacado en alguna materia, si necesita de tutorías, saber dónde estudia (sala, cocina, cuarto), cuánto tiempo le dedica al estudio, si cuenta con apoyo en la casa, entre otras cosas. A partir de este punto se realiza un seguimiento del deportista, asesorando y orientando en los casos que se requiera.

Esfera social. En esta área se pretende hacer un acompañamiento al proceso de diseño del proyecto de vida. Es de suma importancia que tenga claro donde se ve a corto, mediano y largo plazo, no solo como futbolista, sino como miembro de una familia, como estudiante y como profesional académico. Esto colabora a prepararlos mejor para el día del retiro en el deporte. Se les brinda charlas con expertos en diversos campos como: finanzas, nutrición, mercadeo, entre otros. Por otra parte, se debe identificar quiénes son sus pares, sus amigos más cercanos, saber con quién comparte su tiempo de recreación, qué le gusta hacer, qué edades tienen las personas con las que interactúa, si estos son deportistas o no, si le apoyan en el deporte o lo censuran.

En suma, es pertinente saber cómo se encuentran sus relaciones afectivas, que en edades de adolescencia son muy irregulares y tienen un peso importante. En la categoría sub15 y sub-17, la necesidad de aprobación social se encuentra en gran apogeo, lo cual significa que es de suma importancia pertenecer a un grupo y ser acepado sin problemas. Cuando se trabaja con deportistas jóvenes, los compañeros de equipo, los amigos, la pareja afectiva y la familia suelen jugar un papel importante y de gran influencia en formación de la fortaleza mental de futbolista de élite. Es una edad en que las personas son más susceptibles de ser influenciadas, más vulnerables a ser impresionadas y donde existen mayores probabilidades de que las experiencias que vivan y las lecciones que aprendan les dejen una marca para toda su vida (Roffé y Rivera, 2014).

Por otra parte, es relevante tener claro cuáles son las personas más influyentes en ellos, para bien y para mal. Este factor es determinante para que un jugador Ilegue a disfrutar de las mieles del éxito o no. El tiempo para amigos, para ocio, por lo general es limitado, pero debe de tenerlo. Está claro que el desarrollo social del futbolista, no es igual al de resto de chicos que no tienen como proyecto de vida convertirse en un baluarte deportivo.

\section{El trabajo con equipos}

La intervención a nivel de equipo se lleva a cabo cuando el entrenador lo considera necesario, o bien cuando el psicó- 
logo lo solicita y cuenta con la autorización del entrenador, siguiendo los mismos principios de intervención (durante fases preparatorias, eliminatorias y mundiales), mantenimiento o prevención (fase preparatoria y partidos amistosos) mencionados anteriormente. Estas sesiones pueden ser por medio de charlas y/o talleres en donde se utilizan presentaciones, pizarra, videos, entre otros, o bien sesiones de campo para cuando se implementan actividades de trabajo en equipo, concentración, comunicación, etc. Es clave que estas sesiones sean participativas, lúdicas y dinámicas. Lejos de tratarse de una clase magistral típica de universidad, llena de tecnicismo y complejidades, debe ser un espacio en donde se ponga en manifiesto de una manera creativa y amena las variables psicológicas que se considera importantes intervenir. En cuanto la duración puede ser de $30 \mathrm{mi}-$ nutos a 1 hora como máximo.

Estas sesiones se deben planificar con los cuerpos técnicos previamente y deben contar con la aprobación del entrenador. En la mayoría de sesiones pueden estar presentes miembros del cuerpo técnico y entrenador, mientras que en otras solo está presente el psicólogo. Esto porque al estar presente el entrenador $u$ otro miembro, dependiendo del tema en discusión, puede crear resistencias que impide que fluya la palabra o bien su lenguaje no verbal de manera natural, y se ve limitada la retroalimentación. Para ello, debe de quedar muy claro las razones para el entrenador, y no crear mal entendidos que refuercen mitos alrededor del psicólogo del deporte como que "desea adueñarse del vestuario". Si el entrenador no queda satisfecho con los argumentos o bien no se siente cómodo, lo ideal sería diseñar otra estrategia que no ocasione distorsionar la relación psicólogo-entrenador que esencial para el éxito en el trabajo mental del equipo, por ejemplo, trabajar con el cuerpo técnico para que ellos gestionen al equipo.

\section{Servicios médicos}

El psicólogo del deporte es un apoyo más del grupo interdisciplinar de servicios médicos que colabora primordialmente en el trabajo de prevención y recuperación de lesiones (Ver Figura 3). Se trabaja muy de cerca con el fisioterapeuta, ya que los jugadores les ofrecen mucha información clave deportiva y extradeportiva. Se trabajan con los futbolistas lesionados aspectos como: adherencia al programa de rehabilitación, motivación, potenciar los grupos sociales que le acompañe en el proceso, planteamiento de metas, visualización, entre otros. En adición, se ha colaborado para que el equipo médico (médico, fisioterapeuta, nutricionista, readaptador físico, masajista y psicólogo) trabaje de manera más cohesionada entendiendo mejor su rol para ser más eficaces en la tarea de cada uno. En suma, se le brinda atención psicológica al personal de los servicios médicos en campos como manejo de relaciones afectivas, familia, estrés laboral, etc.

Es importante destacar que se debe manejar un leguaje en común. Es decir, cada profesional debe conocer un poco de cada área (médica, nutricional, terapia física, psicología, etc.) para entender mejor los procedimientos y por ende darle un mejor servicio al deportista. Por otra parte, se llevan a cabo reuniones mensuales en donde se hace retroalimentación de casos dirigidos con el fin de mejorar y optimizar el desempeño como equipo de trabajo.

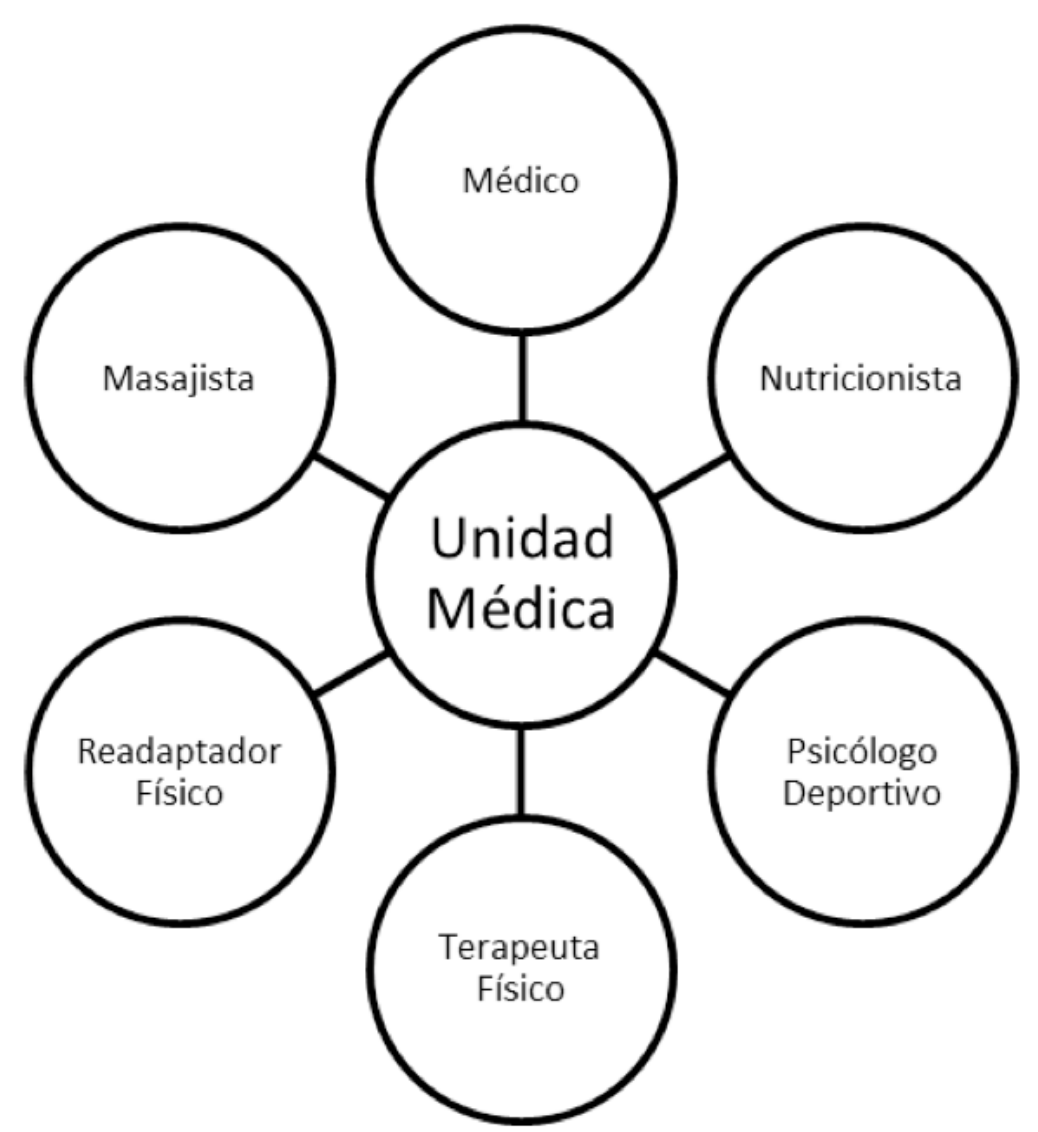

Figura 3. Unidad médica y sus áreas de la Federación Costarricense de Fútbol.

\section{El trabajo con los entrenadores}

Se considera fundamental la colaboración entre psicólogo y entrenador, como lo es entre entrenador y preparador físico, para un buen programa de desarrollo del trabajo psicológico (Olmedilla, García y Garcés, 1998). Si bien es cierto, la labor del psicólogo con los entrenadores es muy versátil, podríamos decir que en primera instancia somos asesores a completa disposición del entrenador y del resto del cuerpo técnico.

¿Qué aspectos se asesoran? Básicamente los relacionados con la mejora de la gestión de los deportistas (e.g., co- 
municación, motivación, modificación de conductas, etc.) y los de autogestión -e.g., regulación emocional, autoliderazgo, etc.- (García-Naveira y Jerez, 2012).

Debe de dejarse en claro que el secreto profesional es clave para que el trabajo sea exitoso. En algún momento, se llevaron a cabo charlas mensuales de temas de interés como: técnicas de negociación, psicología de grupos, liderazgo, etc., para que pudieran incorporar en entrenamientos y competiciones.

También, la formación de los entrenadores de fútbol es uno de los aspectos que ha ido adquiriendo importancia a lo largo de los años (Olmedilla, Ortín, Andréu y Lozano, 2004). Es por ello que, además, se participa como profesor para entrenadores de futbol en la materia Psicología del Deporte de la Licencia B (permite ser entrenador y asistente en segunda división) y Liderazgo Deportivo de la Licencia A (permite ser entrenador y asistente en primera división). El objetivo es facilitar herramientas psicológicas a las nuevas generaciones de entrenadores, y que el rol del psicólogo del deporte les resulte más familiar. Que conozcan los alcances y limitaciones que representa tener un psicólogo en el cuerpo técnico.

\section{Discusión}

La labor como psicólogo del deporte de selecciones nacionales menores de futbol, conlleva a una responsabilidad y reto constante. Se debe de contar con una alta resistencia mental y tolerancia a la frustración, ya que a diferencia de los clubes, es un contexto bastante cambiante e incierto, como por ejemplo el tiempo que se cuenta para trabajar con los jugadores a nivel individual, la rotación de los jugadores debido a irregularidad de rendimiento, las lesiones de los jugadores, situación académica, aspectos económicos, o bien por decisión técnica. También ha pasado que en una concentración se trabaja con un seleccionado, y a la siguiente concentración este ya no está convocado.

En la misión de potenciar las habilidades psicológicas del seleccionado, se tiene que lidiar con el factor tiempo, que tiene que ver con la naturaleza de una selección (reclutar jugadores de concentración en concentración o de torneo en torneo), en el que se trabaja con márgenes muy cortos de tiempo, y el área psicológica no es la excepción. Inclusive, en ocasiones el trabajo queda inconcluso por esta cuestión. Es por ello que dentro de las competencias del psicólogo del deporte debe de estar poseer una alta capacidad de adaptación al cambio y tener mucha perseverancia en su trabajo.

Además, el psicólogo del deporte debe ser muy hábil en sus relaciones interpersonales, ya que está en constante interacción con los cuerpos técnicos y equipos, donde cada entrenador establece su cultura, reglas y códigos a los que se debe adaptar e integrar rápidamente.

En el trabajo con los entrenadores es importante contribuir a su formación para que estos sean mejores profesionales. Por ejemplo, las habilidades sociales de los entrenadores juegan un papel determinante. Estos deben de mejorar la cantidad y la calidad de los mensajes y el feedback que le dan al jugador. El entrenador tiene que ser un educador y un guía, facilitando el crecimiento personal y deportivo del jugador. No tiene que generar falsas expectativas o ser un freno en la proyección del jugador. Tanto el futbolista como entrenador deben estar en completa sintonía, con una perspectiva realista y ambiciosa.

Otro aspecto a tomar en cuenta para el psicólogo del deporte es poseer un conocimiento profundo de las etapas psico-sociales del preadolescente, adolescente y adulto temprano, y como estas interactúan con el mundo del deporte. Es necesario adaptarse al lenguaje que manejan estas poblaciones para poder crear una empatía genuina. Ya que esto último es una de las llaves del éxito.

En otro orden de cosas, también es pertinente destacar que de acuerdo a lo observado en estos años, los seleccionados que poseen una fortaleza mental destacable, y que prevalecen en la élite, se caracterizan por mantener una búsqueda perpetua de la excelencia, por tener un alto grado de auto-motivación, es decir, no es necesario que lo estén reconociendo a cada instante para que rinda al máximo, sabe distinguir entre el resultado del partido, sea perder ganar o empatar y evaluar su propio rendimiento, siempre está dispuesto a aprender de sus errores y sabe cómo hacerle frente a la crítica, es positivo pero realista, posee gran perseverancia, nunca pierde la esperanza, nunca se rinde, logra regular sus emociones, en los momentos de presión es cuando se muestra más seguro y tranquilo, la presión la convierte en motivación. Cuando se encuentra en el entrenamiento o en competición su concentración es plena, le caracteriza una auto-confianza fuerte y balanceada, no busca excusas o justificaciones, sino que se responsabiliza por sus acciones y alienta a los demás. En definitiva, el rendimiento ofrecido se caracteriza por su regularidad, no importa el rival, temperatura, afición, marcador, etc.

No todos los futbolistas tienen las características psicológicas para ser seleccionados. Aquí nace el dilema que no todo jugador que tenga un buen rendimiento en el club, debe tener buen rendimiento en la selección. Las exigencias psíquicas son distintas, la capacidad de adaptación es requisito incuestionable, ya que la presión aumenta considerablemente. Los deportistas también se ven influidos por las expectativas que sobre ellos haya generado la afición, periodistas, familiares y entrenadores. Todas estas son fuentes de infor- 
mación que utilizan para generar los juicios sobre su propia habilidad y la competición por venir. Según Cox (2008), los que poseen destrezas de afrontamiento bien desarrolladas y entrenadas serán más eficaces en el control del estrés y las demandas del entrenamiento y la competición. En este punto los psicólogos del deporte tenemos que incidir en la intervención con el deportista desde una perspectiva formativa y de entrenamiento a medio-largo plazo, para que no haya una gran distancia entre el rendimiento en los clubes y en la selección. En estos casos sería relevante que cada club profesional tuviese un departamento de psicología para que trabajasen con los más jóvenes para conseguir estos y otros objetivos.

También destacar que la identidad del jugador de futbol suele alimentarse del estatus que posea en el medio deportivo. Cuando alcanzan ser seleccionados, ascienden en las posiciones jerárquicas sociales del futbol, de la comunidad, del país. El jugador debe ser lo suficientemente estable emocionalmente para asimilar estepas de transición bruscas (desconvocatorias tempranas) o bien la continuidad en el tiempo con la selección sin caer en zona de confort o en terreno de triunfalismo, que suele atentar en contra del rendimiento y a la postre de la proyección del jugador.

Como mejora, para potenciar el trabajo del psicológico en la federación, se podría ampliar el número de personal que trabaja en el área, como por ejemplo, contando con un especialista en psicología educativa para que les pueda facilitar técnicas de estudio, mantener dialogo constante con los padres de familia e institución académica, y trabajadora social para que se pueda visitar y recopilar información de la realidad donde habita el jugador y así comprender más sus costumbres, creencias y conductas.

Como estrategia de evaluación y seguimiento de la labor del psicólogo del deporte, se recomienda llevar una bitácora diaria de los acontecimientos más relevantes de los jugadores y entrenadores, tanto de los entrenamientos como de la competición. Cualquier detalle deportivo y/o comportamental es útil anotarlo para poder comprender e identificar patrones de conductas individuales y/o grupales.

Como perspectiva futura se quiere diseñar un instrumento para medir las habilidades psicológicas y emocionales de los entrenadores de selecciones regionales. Estos sujetos llevan a cabo un papel fundamental, ya que son el primer contacto que tiene el jugador para iniciar un largo proceso para ser seleccionado nacional. El objetivo es detectar las fortalezas y aéreas de mejora del entrenador para asegurarse que ese primer paso lo manejen entrenadores lo mejor capacitados posible.

En definitiva, ser psicólogo del deporte, quien trabaja con selecciones de futbol, es una gran oportunidad para crecer en el ámbito personal y profesional. En ocasiones, se tiene la sensación que es más lo que se aprende que lo que se facilita. Trabajar con seleccionados es un gran honor y privilegio, ya que se está trabajando con un sector especial de la sociedad, con individuos que poseen grandes habilidades técnicas, físicas, pero sobre todo, poseen una gran calidad humana.

El trabajo del psicólogo se encuentra finalizado, no solo cuando clasifican a un mundial o bien avanzan fases de grupos durante mundiales, sino cuando se convierten en jugadores de alto rendimiento. Lo maravilloso de la Psicología del Deporte es que todas las estrategias enseñadas para combatir adversidad y rendir al máximo nivel, son extrapolables a la vida cotidiana, sea cuando se conviertan en eventuales padres de familia, profesionales, amigos, estudiantes, etc. Lo que confirma que se está en la profesión correcta, es cuando se ve a los deportistas triunfando en la vida, y aun mejor cuando valoran y ofrecen muestras de agradecimiento al trabajo realizado.

\section{Referencias}

Cantón, E. (2010). La Psicología del Deporte como profesión especializada. Papeles del Psicólogo, 37, 237-245.

Colegio Oficial de Psicólogos de España [COP] (1998). Perfiles profesionales del psicólogo. Madrid, España: Autor.

Cox, R. (2008). Psicología del Deporte Conceptos y sus aplicaciones (Sexta edición). Madrid, España: Medica Panamericana.

Díaz Morales, J. F. y García Naveira, A. (2001). Evaluación de metas en jugadores de fútbol de categoría juvenil y absoluta. Revista de Psicología del Deporte, 10, 211-224.

Federación Costarricense de Futbol (s.f. a). Historia del futbol en Costa Rica. Recuperado

de http://fedefutbolcr.com/historia-del-futbol-en-cr/

Federación Costarricense de Futbol (s.f. b). Comité ejecutivo. Recuperado de http://fedefutbolcr.com/comite-ejecutivo/

García-Naveira, A. (2010). El psicólogo del deporte en el alto rendimiento: aportaciones y retos futuros. Papeles del Psicólogo, 31, 143-154.

García-Naveira, A. y Jerez, P. (2012). Departamento de Psicología del Club Atlético de Madrid: filosofía, programación y desempeño profesional en el fútbol base. Cuadernos de Psicología del Deporte, 12(1), 111-120.

Gould, D. y Petlichkoff, L. (1988). Participation motivation and attrition in young athletes. En F. Smoll, R. Magill y M. Ash (Eds.), Children in Sport (3 ${ }^{\mathrm{a}}$ ed., pp. 161-178). Champaign, IL, USA: Human Kinetics.

Holt, N.L. y Dunn, J.G. (2004). Toward a Grounded Theory of the psychosocial competencies and Environmental conditions associated with soccer success. Journal of Applied Sport Psychology, 16, 199-219.

Marí, P. (2011). Aprender de los campeones. Barcelona, España: Plataforma Editorial.

Revista de Psicología Aplicada al Deporte y al Ejercicio Físico (2016), 1, e5, 1-10 
Miller, P.S. y Kerr, G. (2002). Conceptualizing excellence: Past, Present and Future.

Journal of Applied Sport Psychology, 14, 140-153.

Olmedilla, A., García, C. y Garcés, E. (1998). Un análisis del papel profesional del psicólogo del deporte desde la percepción del entrenador de fútbol. Revista de Psicología del Deporte, 7, 95113.

Olmedilla, A., Ortín, F., Andréu, M. y Lozano, F. (2004). Formación en psicología para entrenadores de fútbol: una propuesta metodológica. Revista de Psicología del Deporte, 13, 247-262.
Roffé, M. (2008). Psicología del jugador de futbol: con la cabeza hecha pelota. Buenos Aires, Argentina: Lugar Editorial.

Roffé, M. (2009). Evaluación psicodeportologica: los 30 test psicométricos y proyectivos. Buenos Aires, Argentina: Lugar Editorial.

Roffé, M. y Rivera, S. (2014). Entrenamiento mental en el futbol Moderno. Madrid, España: Futbol de Libro.

Weinberg, A. y Gould, D. (2010). Fundamentos de psicología del deporte y del ejercicio físico. (4 Ed.) Madrid, España: Médica Panamericana. 